\title{
0 desenvolvimento da proficiência motora em crianças ciganas e não ciganas: um estudo comparativo.
}

José Francisco Filipe Marmeleira ${ }^{1}$ e João Paulo Abreu ${ }^{1}$

${ }^{1}$ Universidade de Évora, Portugal

Francisco, J.; Marmeleira, F.; 0 desenvolvimento da proficiência motora em crianças ciganas e não ciganas: um estudo comparativo. Motricidade 3(1): 289-297

\section{Resumo}

O objectivo deste trabalho foi o de estudar possíveis influências da etnia sobre o desenvolvimento motor. Para tal foi constituída uma amostra de 60 crianças de ambos os géneros com 7, 8 e 9 anos de idade: 30 crianças ciganas que frequentavam uma escola do $1^{\circ}$ ciclo em Moura; 30 crianças não ciganas que frequentavam uma escola do $1^{\circ}$ ciclo em Lisboa. Para estudo e comparação dos níveis de desenvolvimento motor dos dois grupos da amostra, foi utilizada a forma reduzida do Teste de Proficiência Motora de BruininksOseretsky. Concluiu-se que as crianças não ciganas em comparação com crianças de etnia cigana, apresentavam valores significativamente superiores da motricidade global $(\mathrm{p}=0,015)$, da motricidade fina $(p=0,000)$ e da própria proficiência motora $(p=0,005)$.

Palavras-chave: proficiência motora, meio sócio-cultural, etnia cigana

Data de submissão: Março 2006

Data de aceitação: Dezembro 2006

\section{Abstract}

The motor proficiency development in gypsy and non-gypsy children: a comparative study

The objective of this study was to investigate possible influences of ethnicity on motor development. The sample comprised 60 children of both genders with 7,8 and 9 years of age: 30 gypsy children who attended an elementary school in Moura; 30 non-gypsy children who attended an elementary school in Lisboa. The short form of the Bruininks-Oseretsky Test of Motor Proficiency was used to study and compare the motor development level of the sample groups. We concluded that the non-gypsy children in comparison to the gypsy children, revealed significative superior results in gross motor performance $(p=0,015)$, fine motor performance $(p=0,000)$ and in motor proficiency $(\mathrm{p}=0,005)$.

Key words: motor development, sociocultural background, gypsies 


\section{Introdução}

Em Portugal vários estudos têm investigado a influência de meios sócio-culturais diferenciados no desenvolvimento motor das crianças, designadamente os efeitos da residência em meio urbano ou meio rural ${ }^{2,17,19,20}$. Há, no entanto, uma lacuna de estudos sobre a influência de outras variáveis sócio-culturais no desenvolvimento motor, entre elas as relacionadas com a etnicidade.

Alguns trabalhos realizados noutros países têm estudado a importância da origem étnica em factores como os padrões de actividade física e/ou a aptidão física ${ }^{8,9,14,16}$. No entanto, e no que se refere à etnia cigana, não foi encontrado qualquer estudo no âmbito do desenvolvimento motor. Provavelmente, tal situação terá a ver com o facto dos ciganos continuarem a ser considerados pelos não ciganos pouco acessíveis, o que se têm traduzido num conhecimento limitado sobre as suas características sócio-culturais ${ }^{24}$.

Em Portugal, a comunidade cigana é constituída por cerca de 50000 indivíduos ${ }^{3}$. Na sua maioria, a mesma é sedentária ou semi-nómada pelo que o número da população itinerante tem vindo a diminuir $^{13}$

A integração escolar da comunidade cigana é muitas vezes difícil e as crianças apresentam uma taxa elevada de insucesso e abandono escolar em Portugal ${ }^{3}$. A maior presença de alunos de etnia cigana verifica-se ao nível do $1^{\circ}$ ciclo $(10,9 \%$ de todos os alunos provenientes de minorias étnicas) 13 .

Entre a comunidade cigana a brincadeira e o jogo parecem ter algumas características particulares. Segundo Levinson ${ }^{7}$ a brincadeira cumpre uma importante função cultural entre a comunidade cigana, preparando as crianças para o futuro e transmitindo-lhes instrumentos indispensáveis ao seu contexto sócio-económico. Segundo o autor, as crianças raramente estão sozinhas, passando muito tempo integradas em grupos de idades diferenciadas, o que tem um impacto marcante no tempo para brincar bem como no tipo de brincadeiras. Refere, ainda, que a realização de actividades intergeracionais é comum durante os momentos recreativos e de trabalho.

Entre os ciganos há muitas vezes uma expectativa de que as crianças devem contribuir desde muito cedo para a família. Tal facto, pode ser considerado uma desvantagem na medida em que determinadas formas de brincar se tornam um luxo inacessível, mas, por outro lado, uma vantagem porque as crianças ciganas podem usufruir de uma posição mais relevante na vida familiar e comunitária que advém das responsabilidades assumidas ${ }^{7}$.

Levinson ${ }^{7}$ refere que encontrou poucos brinquedos entre as crianças ciganas. Relata que observou muitas vezes as crianças a brincar com objectos que na sociedade mais abrangente seriam pouco conotados com a recreação mas mais com o mundo do trabalho. Considera, ainda, que entre a comunidade cigana não existe uma clara distinção entre trabalho e lazer como nas economias modernas.

Thomson e Soós ${ }^{24}$ realizaram um estudo sobre as atitudes dos jovens ciganos em relação ao desporto, em duas instituições de ensino secundário na Hungria especialmente vocacionadas para o ensino da cultura cigana. Concluíram que os estudantes de etnia cigana (i) não apresentavam uma atitude tão positiva em relação ao desporto e à actividade física quanto os outros jovens húngaros, (ii) tinham um nível de prática desportiva fora da escola relativamente baixo, (iii) consideravam a melhoria da saúde e aptidão física como o principal objectivo do desporto, (iv) aceitavam na sua maioria a noção de que muitos desportos são específicos para determinado género.

No presente trabalho, os grupos da amostra viviam em meios com características urbanísti- 
cas muito diferenciadas. Segundo Neto ${ }^{11}$ as alterações ao nível do desenvolvimento motor são mais vincadas nos grandes meios urbanos, onde a procura de um melhor nível de vida leva a uma alteração ou adaptação dos comportamentos e hábitos do quotidiano. Estas alterações são mais visíveis na motricidade infantil, em que as capacidades de mobilidade e exploração corporal na vida das crianças estão comprometidas devido à escassez de espaços ${ }^{11,18}$. De facto, no meio urbano, a densidade habitacional e de tráfego, os estilos de vida da família e a gestão do tempo das crianças, dificultam o seu acesso aos espaços da rua e aos grandes espaços verdes ${ }^{12,22}$.

Alguns estudos têm comparado a performance motora em função do meio (urbano versus rural), tendo sido encontrados resultados significativamente superiores entre crianças do meio rural na coordenação geral $^{19,20}$ e no lançamento em distância ${ }^{20,21}$. Apenas um estudo ${ }^{19}$ refere um item onde registou performances significativamente superiores das crianças que habitavam no meio urbano, no caso a coordenação fina.

Moreno \& Vasconcelos ${ }^{10}$ encontraram valores significativamente superiores nas provas de dinamometria manual e de corrida com mudança de direcção entre adolescentes do sexo feminino que habitavam no meio rural

Costa $^{4}$ encontrou várias diferenças estatisticamente significativas num estudo comparativo do desenvolvimento motor de crianças oriundas de países africanos de expressão portuguesa com o de crianças caucasianas de nacionalidade portuguesa: as primeiras tinham performances superiores nas provas de equilíbrio, de força inferior, média e superior; as segundas apresentavam valores superiores na agilidade e coordenação.

Alguns estudos realizados noutros países compararam a aptidão física de grupos de crianças ou jovens provenientes do meio rural e urbano. Ozdirenc et al. ${ }^{15}$ encontraram níveis significativa- mente inferiores de flexibilidade e de resistência muscular entre as crianças que residiam em áreas urbanas; Taks et al. ${ }^{23}$ não encontraram diferenças entre raparigas adolescentes; Tsimeas et al. ${ }^{23}$ concluíram que o local de residência não tem um impacto marcante na aptidão física das crianças.

Quanto à prática desportiva, alguns estudos indicam que a mesma é significativamente superior entre as crianças e jovens que habitam em zonas urbanas ${ }^{14,23}$. Também foram encontrados maiores níveis de performance motora entre crianças com maior estatuto sócio-económico ${ }^{6,14}$ e que participavam em desportos fora da escola ${ }^{14}$.

Neste contexto, o presente estudo pretendeu estudar a influência da etnia sobre o desenvolvimento motor. Deste modo, procedeu-se à comparação da proficiência motora de um grupo de crianças ciganas com a de um grupo de crianças não ciganas.

\section{Metodologia}

\section{Amostra}

A amostra foi constituída por 60 crianças com idades compreendidas entre o 7 e os 9 anos, constituída por dois grupos com idades similares: 30 crianças ciganas (16 rapazes e 14 raparigas) que frequentavam a Escola Básica do $1^{\circ}$ ciclo, $N^{\circ} 3$ de Moura; 30 crianças não ciganas (15 rapazes e 15 raparigas) que frequentavam a Escola Básica do $1^{\circ}$ ciclo, N. ${ }^{\circ} 111$ de S. João de Brito, no centro da cidade de Lisboa. O quadro n. ${ }^{\circ} 1$ faz uma pequena caracterização da amostra. 
0 desenvolvimento da proficiência motora em crianças ciganas e não ciganas: um estudo comparativo.

José Francisco Filipe Marmeleira e João Paulo Abreu

\begin{tabular}{lccc}
\hline \multicolumn{2}{c}{ Quadro n. ${ }^{0}$ 1: Caracterização dos grupos } & & \\
\hline Grupo & $\begin{array}{c}\text { Idade em anos } \\
\text { (Média } \pm \text { DP) }\end{array}$ & N. ${ }^{\circ}$ de crianças por & Meio \\
Crianças ciganas & $8,33 \pm 0,89$ & género & Moura \\
Crianças não ciganas & $8,00 \pm 0,59$ & 16 M., $14 \mathrm{~F}$. & Lisboa \\
\hline
\end{tabular}

As crianças de etnia cigana habitavam em barracas nos arredores da cidade de Moura (Alentejo interior), num meio geográfico em que dispunham de um grande espaço físico livre onde referiram realizar diversas actividades lúdicas (jogo da apanhada, jogo das escondidas, jogar à bola, etc.). Uma parte substancial do seu tempo livre era ocupada com diversas actividades relacionadas com a ajuda aos mais velhos na realização de tarefas da vida diária. Esta caracterização vai de encontro ao referido por Levinson ${ }^{7}$, já citado na revisão bibliográfica.

Por sua vez, as crianças não ciganas (caucasianas com excepção de uma criança), habitavam num meio urbano, caracterizado pela grande limitação de espaços livres, fazendo com que as suas brincadeiras se confinassem mais a pequenos largos, ao passeio, ou ao próprio interior da casa (jogos de computador, consolas, ver televisão, etc.).

Quanto à prática de actividades físico-desportivas no âmbito escolar, o grupo de crianças não ciganas que vivia em Lisboa, tinha Educação Física (EF) duas vezes por semana sob a orientação de um professor da especialidade. $\mathrm{O}$ grupo de crianças ciganas que vivia em Moura não chegava a ter, em média, uma aula de EF por semana, a qual era leccionada pelo próprio professor titular. Fora do âmbito escolar, 23 crianças não ciganas referiram praticar actividades desportivas regularmente (2 a 3 vezes por semana), na sua maioria natação, ginástica e futebol.

Nenhuma criança de etnia cigana referiu a prática regular de actividades físicas desportivas.

\section{Procedimentos}

Neste trabalho, para efeito comparativos do nível de desenvolvimento motor dos grupos da amostra, foi utilizado o Teste de Proficiência Motora de Bruininks-Oseretsky (TPMBO) na sua forma reduzida.

A aplicação do TPMBO decorreu durante uma semana em cada uma das escolas seleccionadas, tendo sido utilizados para o efeito espaços cobertos e fechados. A amostra foi distribuída em subgrupos de 3 ou 4 crianças, as quais foram avaliadas individualmente em cada uma das provas. Em ambas as escolas metade das crianças realizaram as provas durante o horário escolar da manhã e a outra metade durante o horário escolar da tarde. A forma reduzida do TPMBO estuda três componentes da proficiência motora: motricidade global, composta e fina. No total são realizadas 14 provas (itens) que integram 8 sub-testes: corrida de velocidade/agilidade, equilíbrio, coordenação bilateral, força, coordenação dos membros superiores, velocidade de reacção, visuomotricidade e dextralidade. De referir que Bruininks ${ }^{1}$ define proficiência motora como um termo genérico que se refere à performance obtida numa vasta gama de testes motores.

Os valores obtidos em cada um dos itens foram convertidos em pontos segundo o manual do TPMBO $^{1}$. Os valores da motricidade fina, global e composta, resultaram da soma da pontuação obtida nos itens que as constituem. Os valores de proficiência motora resultaram da soma da pontuação de todos os itens.

O TPMBO foi desenvolvido para estudar as 
aquisições motoras de crianças e jovens, não só avaliando funções e disfunções motoras, como inclusivamente atrasos de desenvolvimento. A sua aplicação pode ser feita em crianças entre os 4,5 e o 14,5 anos ${ }^{1}$. É um teste credível e de ampla aplicação, quer na psicologia, quer na educação ${ }^{5}$.

\section{Estatística}

Para efeitos de comparação entre os grupos da amostra, e uma vez que não se verificou a normalidade da distribuição dos resultados na grande maioria das variáveis estudadas, procedeu-se à utilização do teste não paramétrico de MannWhitney. Foi utilizado um nível de significância de $\mathrm{p}<0,05$.

O tratamento estatístico foi efectuado através do programa SPSS para Windows, Copyright SPSS ${ }^{\circ}$ Inc., versão 13.0

\section{Resultados}

Foram encontradas diferenças estatisticamente significativas entre os resultados obtidos pelos grupos da amostra em muitas das variáveis da proficiência motora. Os resultados encontramse no Quadro $n^{\circ} 2$, destacando-se, desde logo, o facto da amostra de crianças ciganas apresentar valores significativamente inferiores para dois dos três grupos de habilidades analisadas: motricidade global $(p=0,015)$ e motricidade fina $(p=0,000)$.

Ao efectuar-se uma análise por item, verificaram-se na Motricidade Global melhores desempenhos das crianças não ciganas no equilíbrio $(\mathrm{p}=0,00$ para o equilíbrio unipedal) e na corrida de agilidade $(p=0,021)$. Na Motricidade Fina as crianças não ciganas revelaram um controlo visuo-motor superior, mais concretamente no desenho da linha $(\mathrm{p}=0,035)$ e na cópia de figuras ( $\mathrm{p}=0,000$ para cópia de lápis sobrepostos). Apresentaram, ainda, maior destreza manual na marcação de pontos $(\mathrm{p}=0,000)$.

\section{NS: Não Significativo}

Em todos os outros itens não foram encontradas diferenças estatisticamente significativas, no entanto, e à excepção de um teste da motricidade composta (lançamento da bola a um alvo) e de um teste de motricidade fina (velocidade de resposta), as crianças não ciganas obtiveram sempre resultados médios iguais ou superiores aos das crianças ciganas.

$\mathrm{Na}$ sequência dos dados já apresentados, o nível global de Proficiência Motora foi significativamente superior $(\mathrm{p}=0,005)$ entre o grupo de crianças não ciganas.

\section{Discussão}

Em alguns estudos têm sido referidas diferenças em determinadas variáveis do desenvolvimento motor das crianças, em função da proveniência geográfica - meio rural versus meio urbano $15,19,20,21$. No entanto, os resultados apontam uma tendência oposta ao verificado neste estudo, i.e., quando detectadas diferenças as crianças que vivem em meio rural estão, frequentemente, numa posição vantajosa. Para explicar tal facto têm sido apontados factores como a acessibilidade aos espaços para brincar, as características dos mesmos, e os estilos de vida relacionados com a gestão do tempo de trabalho e de lazer ${ }^{12,22}$.

Porque razão as crianças ciganas, que têm maiores vantagens na acessibilidade aos espaços fisicos abertos e livres, apresentam resultados inferiores na performance motora? De um modo geral poucos trabalhos publicados estudaram aprofundadamente a cultura da população cigana, designadamente nos seus hábitos de lazer. No entanto, os que existem e que foram referidos na introdução, apontam para diferenças substanciais na forma como a população de etnia cigana entende o lazer e a própria brincadeira. Esta parece ter, desde cedo, um papel característico 
$\mathbf{O}$ desenvolvimento da proficiência motora em crianças ciganas e não ciganas: um estudo comparativo.

José Francisco Filipe Marmeleira e João Paulo Abreu

Quadro n. ${ }^{0}$ 2: Pontuação (Média \pm DP) obtida pelo grupo de crianças ciganas e pelo grupo de crianças não ciganas nos itens avaliados pela forma reduzida do TPMBO. Valores significativos de p para a comparação entre grupos obtido através do teste Mann-Whitney.

\begin{tabular}{|c|c|c|c|}
\hline & $\begin{array}{c}\text { Crianças } \\
\text { ciganas }\end{array}$ & $\begin{array}{c}\text { Crianças } \\
\text { não ciganas }\end{array}$ & $\mathrm{p}$ \\
\hline Motricidade Global & $24,73 \pm 4,72$ & $27,60 \pm 3,05$ & 0,015 \\
\hline Corrida de Agilidade & $8,47 \pm 1,31$ & $9,30 \pm 1,15$ & 0,021 \\
\hline Equilíbrio Unipedal: equil. sobre uma trave Equilíbrio & $4,30 \pm 1,75$ & $5,67 \pm 1,06$ & 0,000 \\
\hline Dinâmico: andar sobre uma trave & $2,83 \pm 1,49$ & $3,17 \pm 1,09$ & NS \\
\hline Coordenação Bilateral: batim MI c/ círculos MS & $0,13 \pm 0,35$ & $0,13 \pm 0,35$ & NS \\
\hline Coordenação Bilateral: salto c/ palmas & $1,97 \pm 0,62$ & $2,27 \pm 0,52$ & NS \\
\hline Força: salto em comprimento sem balanço & $7,03 \pm 2,13$ & $7,07 \pm 1,36$ & NS \\
\hline Motricidade Composta & $4,73 \pm 1,36$ & $4,47 \pm 1,04$ & NS \\
\hline Coordenação Manual: agarrar uma bola & $2,43 \pm 0,94$ & $2,43 \pm 0,68$ & NS \\
\hline Coordenação Manual: lanç.bola ao alvo & $2,30 \pm 0,79$ & $2,03 \pm 0,77$ & NS \\
\hline Motricidade Fina & $17,87 \pm 3,92$ & $21,47 \pm 2,80$ & 0,000 \\
\hline Velocidade de resposta & $4,90 \pm 2,43$ & $4,77 \pm 1,22$ & NS \\
\hline Controlo Visuo-Motor: desenhar uma linha & $3,17 \pm 1,15$ & $3,63 \pm 0,72$ & 0,035 \\
\hline Controlo Visuo-Motor: copiar círculos & $1,17 \pm 0,59$ & $1,60 \pm 0,56$ & NS \\
\hline Controlo Visuo-Motor: copiar lápis sobrepostos & $0,43 \pm 0,68$ & $1,27 \pm 0,87$ & 0,000 \\
\hline Dextralidade: distribuir cartas & $3,80 \pm 1,13$ & $4,33 \pm 1,16$ & NS \\
\hline Dextralidade: marcar pontos & $4,40 \pm 1,00$ & $5,87 \pm 1,20$ & 0,000 \\
\hline Proficiência Motora & $47,33 \pm 8,93$ & $53,53 \pm 5,70$ & 0,005 \\
\hline
\end{tabular}

na preparação das crianças para o futuro, transmitindo-lhes instrumentos indispensáveis ao seu contexto sócio-económico.

Segundo Levinson ${ }^{7}$ as crianças ciganas estão muitas vezes em grupos de idades diferenciadas, passam pouco tempo sozinhas e utilizam poucos dos brinquedos típicos da sociedade moderna. $\mathrm{Na}$ opinião do autor, entre a etnia cigana não existe uma distinção muito vincada entre trabalho e lazer como sucede nas economias modernas. Levinson \& Sparkes (2003) citados por Levinson ${ }^{7}$ referem que as crianças agem como adultos desde muito jovens para ganhar estatuto social.

Todas estas especificidades estabelecem distinções óbvias com a maioria das crianças não ciganas, pelo que, mesmo na escola, é comum encontrarem-se nos recreios as crianças ciganas separadas das outras crianças, muitas vezes por sua 
própria opção ${ }^{7}$. Comum entre muitas das crianças ciganas é a ideia de que o conhecimento aprendido em casa é mais importante do que aquele aprendido na escola ${ }^{7}$.

Outra diferença substancial que pode estar na origem das diferenças encontradas, prende-se com o facto das crianças não ciganas terem um acesso mais facilitado à educação físico-motora e a vivências desportivas multidisciplinares, o que pode potenciar um maior desenvolvimento motor.

$\mathrm{Na}$ amostra deste estudo, essas diferenças situavam-se não apenas em termos de EF escolar mas também em termos de actividades para além do espaço escolar. É importante relembrar que os alunos ciganos tinham, em média, menos de uma aula de EF por semana (dada pelo próprio professor titular), enquanto as outras crianças tinham EF duas vezes por semana sob a orientação de um professor licenciado na Área. Fora do âmbito escolar, a maioria das crianças não ciganas $(76,7 \%)$ praticavam actividades desportivas regularmente (2 a 3 vezes por semana), enquanto nenhuma criança de etnia cigana o fazia. $\mathrm{O}$ facto da maioria das crianças do meio urbano praticarem desporto nos seus tempos livres, vai de encontro ao referido por $\mathrm{Neto}^{12}$, de que a crescente institucionalização dos tempos livres das crianças das cidades se tem tornado uma alternativa ao jogo livre e espontâneo

É importante salientar que, como consequência do abandono escolar precoce de muitas crianças ciganas, o acesso a actividades fisico-desportivas praticadas na escola e mesmo fora desta, está bastante comprometido. Por exemplo, no $3^{\circ}$ ciclo do ensino básico e, sobretudo, no ensino secundário, a presença de jovens de etnia cigana é quase inexistente ${ }^{13}$.

Vários estudos referem que o nível de prática desportiva é inferior entre as crianças que habitam num meio rural ${ }^{12,14,23}$ e entre crianças com um estatuto sócio-económico inferior ${ }^{6,14}$.Ambos os factores são, de algum modo, característicos das crianças do grupo de etnia cigana, o que também ajuda a explicar os valores nulos de prática desportiva regular.

A relação da etnia cigana com o desporto carece também de investigação. No único estudo encontrado sobre o assunto, Thomson e Soós ${ }^{24}$ referem que, entre os jovens húngaros de etnia cigana que frequentavam duas escolas secundárias (com uma maioria de alunos de etnia cigana), existia uma atitude menos positiva em relação ao desporto e à actividade física que entre os outros jovens húngaros. Referem, ainda, que os mesmos tinham um nível de prática desportiva fora da escola relativamente baixo.

A temática abordada neste trabalho permitiu levantar algumas questões que seria importante aprofundar em futuras investigações no âmbito do desenvolvimento motor da comunidade de etnia cigana. Por exemplo, seria pertinente estudar o nível de desenvolvimento motor em função da idade biológica das crianças; do mesmo modo, seria importante caracterizar de forma mais sistematizada as actividades desenvolvidas pelas crianças ciganas no seu tempo de lazer.

Neste estudo, procedeu-se à comparação do desenvolvimento motor de crianças ciganas com o de crianças não ciganas. Destacam-se as seguintes conclusões:

- Quando comparadas com crianças ciganas, as crianças não ciganas apresentam valores significativamente superiores na motricidade global, na motricidade fina e na proficiência motora.

- As mesmas diferenças significativas são verificadas para diversos sub-testes da bateria utilizada: corrida de agilidade, equilíbrio unipedal, dextralidade - marcação de pontos, controlo visuomotor - cópia de lápis sobrepostos e desenho de uma linha.

- $\mathrm{O}$ acesso privilegiado das crianças nas cidades a 
$\mathbf{O}$ desenvolvimento da proficiência motora em crianças ciganas e não ciganas: um estudo comparativo.

José Francisco Filipe Marmeleira e João Paulo Abreu

\section{Referências}

práticas físico-motoras devidamente estruturadas e regulares (educação física e desporto extra-curricular) poderá ser um dos factores mais importantes na origem das diferenças encontradas.

- Outro factor poderá ser a especificidade sóciocultural da população de etnia cigana, que se traduz num estilo de vida com características diferenciadas designadamente no que diz respeito aos hábitos de lazer.

\section{Correspondência}

José Marmeleira.

Rua Marechal Francisco da Costa Gomes, lote 1 ,

7080-019Vendas Novas

jmarmel@uevora.pt
1. Bruininks R. (1978). Bruininks-Oseretsky Motor Proficiency Test (BOMPT): Examiner's manual. Minnesota: American Guidance Services.

2. Carvalho A. (1994). Desenvolvimento, capacidades motoras e rendimento motor: a influencia dos contextos rural e urbano. Dissertação de Mestrado não publicada. Lisboa: Faculdade de Motricidade Humana.

3. Comissão Europeia contra o Racismo e a Intolerância (2002). Segundo relatório sobre Portugal. Estrasburgo: Conselho da Europa.

4. Costa A. (1991). Desenvolvimento motor: análise comparativa de dois grupos étnicos, masculinos e femininos, de crianças dos 7 aos 9 anos. Boletim da Sociedade Portuguesa de Educação Física. 2-3:139-158.

5. Fonseca V, Dinis A, Moreira N. (1994). Proficiência em crianças normais e com dificuldades de aprendizagem: estudo comparativo e correlativo com base no Teste de Proficiência Motora de Bruininks-Oseretsky. Rev Ed Esp Reabilitação. 2:7-41.

6. Krombholz H. (1997) Physical performance in relation to age, sex, social class and sports activities in kindergarten and elementary school. Perceptual and Motor Skills. 84(3 Pt 2):1168-70. 7. Levinson MP. (2005). The role of play in the formation and maintenance of cultural identity: gypsy children in home and school contexts. J Cont Ethnography. 34(5):499-532.

8. McKenzie TL, Sallis JF, Elder JP, Berry CC, Hoy PL, Nader PR, Zive MM, Broyles SL. (1997). Physical activity levels and prompts in young children at recess: a two-year study of a bi-ethnic sample. Res Quart Exerc and Sport. 68(3):195-202.

9. McKenzie TL, Sallis JF, Nader PR, Broyles SL, Nelson JA. (1992). Anglo- and Mexican-American preschoolers at home and at recess: activity patterns and environmental influences. J Dev and 
Beh Ped. 13(3):173-80.

10. Moreno D, Vasconcelos O. (2003). Aptidão física, estatuto maturacional e indicadores biossociais: estudo em adolescentes do sexo feminino de dois meios distintos (rural e urbano). In: Congresso Internacional mulheres e desporto: agir para a mudança. Porto.

11. Neto C. (1979).A criança, o Espaço e Desenvolvimento Motor. Ludens. 3(2/3):35-45.

12. Neto C. (2003). Tempo e espaço de jogo para a criança: rotinas e mudanças sociais. In Carlos Neto (ed.), Jogo \& desenvolvimento da criança. Lisboa: edições FMH. p. 10-22.

13. Observatório Europeu para o Racismo e a Xenofobia (2004). Migrants, minorities and education: documenting discrimination and integration in 15 member states of the European Union. Luxembourg: office for official publications of the European Communities.

14. O'Loughlin J, Paradis G, Kishchuk N, Barnett T, Renaud L. (1999). Prevalence and correlates of physical activity behaviors among elementary schoolchildren in multiethnic, low income, inner-city neighborhoods in Montreal, Canada.Ann Epidem. 9(7):394-6.

15. Ozdirenc M, Ozcan A, Akin F, Gelecek N. (2005). Physical fitness in rural children compared with urban children in Turkey. Ped Int. 47(1):26-31.

16. Patrick K, Norman GJ, Calfas KJ, Sallis JF, Zabinski MF, Rupp J, Cella J. (2004). Diet, physical activity, and sedentary behaviors as risk factors for overweight in adolescence. Arc Ped Adol Med. 158(4):385-90.

17. Peixeiro R. (1995). Desenvolvimento das capacidades físicas em crianças dos 6 aos 9 anos de idade no meio rural, misto e urbano: um estudo comparativo. Dissertação de Monografia não publicada. Lisboa: Faculdade de Motricidade Humana.

18. Pimentel J. (1985). Os dados psicológicos: espaço e seu significado no desenvolvimento da criança segundo H. Wallon. Ludens. 9(3):39-43.

19. Pimentel J, Oliveira J. (2003). Influência do meio no desenvolvimento da coordenação motora global e fina: estudo com crianças de 9 e 10 anos da cidade do Porto e da Beira Alta. Horizonte. 18(105):34-37.

20. Pissarra M. (1993). Desenvolvimento motor e envolvimento social: estudo do crescimento e desenvolvimento das capacidades motoras em crianças dos 7 aos 9 anos de idade nos meios rural e urbano. Dissertação de Mestrado não publicada. Lisboa: Faculdade de Motricidade Humana.

21. Serra M. (1992). Desenvolvimento motor, jogo e contexto cultural: estudo comparativo da actividade de crianças com 6,7,8 e 9 anos pertencentes a Meios Socioculturais diferenciados. Dissertação de Mestrado não publicada. Lisboa: Faculdade de Motricidade Humana.

22. Serrano J, Neto C. (2003). As rotinas de vida diária das crianças com idades compreendidas entre os 7 e os 10 anos nos meios rural e urbano. In Carlos Neto (ed.), Jogo \& desenvolvimento da criança Lisboa: edições FMH. p. 206-225.

23. Taks M, Renson R, Beunen G, Claessens A, Colla M, Lefreve J, Ostyn M, Schueremans C, Simons J, Van Gerven D, Vanreusel B. (1991). Sociogeographic variation in the physical fitness of a cross-sectional sample of Flemish girls 13 to 18 years of age. Am J Hum Biol. 3(5):503-513.

24. Thomson RW, Soós I. (2005). Rroma culture and physical culture in hungary. International review for the sociology of sport. 40(2):255-263.

25. Tsimeas, P.D., Tsiokanos, A.L., Koutedakis, Y., Tsigilis, N. \& Kellis, S. (2005). Does living in urban or rural settings affect aspects of physical fitness in children? An allometric approach. Brit J Sports Med. 39(9):671-674. 